\title{
Experimental Assessment of Specific Absorption Rate Using Room Electromagnetics
}

\author{
Aliou Bamba ${ }^{1, *}$, Wout Joseph ${ }^{1}$, member IEEE, Jørgen Bach Andersen ${ }^{2}$, Life Fellow \\ IEEE, Emmeric Tanghe ${ }^{1}$, Günter Vermeeren ${ }^{1}$, David Plets ${ }^{1}$, Jesper Ødum Nielsen ${ }^{2}$ and \\ Luc Martens ${ }^{1}$, member IEEE. \\ ${ }^{1}$ Ghent University / IBBT, Department of Information Technology \\ Gaston Crommenlaan 8 box 201, B-9050 Ghent, Belgium \\ Fax: +3293314 899, Tel: +3293314917 \\ *Email: aliou.bamba@intec.ugent.be \\ ${ }^{2}$ APNet, Department of Electronic Systems \\ Faculty of Engineering and Science, Aalborg University \\ DK-9220 Aalborg, Denmark
}

\begin{abstract}
A closed room environment is viewed as a lossy cavity, characterized by possibly a line of sight (LOS) component and diffuse scattering parts from walls and internal obstacles. A theory used in acoustics and reverberation chambers is applied for the electromagnetics case, and main issues related to measurement systems, antennas characteristics, diffuse energy properties, and human exposure are investigated. The goal of this paper aims first towards validation of the assessment of the reverberation time in an environment using a virtual MIMO channel system. Secondly, the reverberation time in an adjacent room is investigated and hence a measurement-based method is readily developed to assess the absorption cross section and the whole-body specific absorption rate of humans at $2.3 \mathrm{GHz}$ in a realistic closed environment.
\end{abstract}

Index Terms:

Room electromagnetics, reverberation time, absorption cross section, power density, specific absorption rate, SAR, diffuse scattering.

\section{INTRODUCTION}

I NDOOR microwave propagation has been investigated in detail for a long time. More recently, room electromagnetics has become appealing because it does not require full knowledge of all details of the propagation environment [1], which makes it less tricky. Currently, propagation models are complex, [2]-[5]. A very simple propagation model with only few parameters is obtained by considering the indoor environment as a lossy cavity where all the effective losses can be described with a single parameter. The theory of wideband propagation in an environment is applied, similar to studies in acoustics and reverberation chambers. The acoustics community has been applying the method [6] since the $1920 \mathrm{~s}$ (Sabine's equation), but the fundamental difference to the radio case is the polarization [7]. The basic model is very simple and considers a first arriving LOS (Line-Of-Sight) signal if present and after that multiple reflections and scatterings giving rise to a tail with exponential decay and a time constant noted as the reverberation time, similar to the acoustics case. Room electromagnetics basic theory is discussed in [7].

Here the theory of room electromagnetics is applied for transceivers in the same room and for the first time is extended for adjacent rooms.

Electromagnetic measurements near the body are important for electromagnetic compatibility (EMC) studies, for the characterization of antennas near dielectric media, and for potential health effects of electromagnetic radiation.

The reverberation time assessment using a channel sounder is investigated in [1], [7]. In this paper the reverberation time assessment is compared 
for two different measurement systems, namely the channel sounder and the virtual MIMO (Multiple Input Multiple Output) channel sounder. The use of the virtual MIMO has several benefits, i.e., it is much cheaper than the channel sounder, there is no coupling between antennas, etc. However the cost of using a virtual MIMO channel sounder is the increased measurement time, but the measurement of the reverberation time takes only a few minutes. The reverberation time assessment is of main importance because it is a function of the absorption area in the room, the total surface absorbing electromagnetic radiation, and hence linked to the absorption cross section i.e., the body surface area being exposed to radiation, for humans in realistic environments and other lossy objects.

The International Commission on Non-Ionizing Radiation Protection (ICNIRP) [8] defines guidelines for Specific Absorption Rate (SAR) as a basic restriction for human exposure in the Radio-frequency (RF) band. A statistical exposure tool to determine the distribution of the whole-body averaged SAR in realistic indoor-picocell environment is proposed in [9]. The SAR is a difficult quantity to measure in an actual human. Therefor, the studies of the SAR in realistic human body models and realistic environments are based on numerical computations such as the finite-difference time-domain (FDTD) technique, such as in [10], [11]. These computations are dependent on the accuracy of the phantom modeling, the used numerical code, and are time consuming, etc. Rather than using such numerical computational methods, a measurement-based approach based on the absorption cross section (ACS) is a suitable alternative for the assessment of the whole-body averaged SAR $\left(\mathrm{SAR}_{w b}\right)$, and is readily developed in this paper.

From the ACS, and given an incident power density it is hence easy to derive the whole-body SAR [12]. The outline of this paper is as follows: the two measurement systems configuration and the environments are described in Section II. Section III describes the methodology to extract the reverberation time $(\tau)$, in Section IV we present the benchmarking results of both measurement systems and other features of the reverberation time. Section V is devoted to the absorption cross section assessment and the experimental whole-body SAR calculation.
Conclusions are presented in Section VI.

\section{CONFIGURATION AND ENVIRONMENT}

\section{A. Virtual MIMO channel sounder setup}

The virtual channel sounder setup for the MISO (Multiple Input Single Output) measurements is shown in Fig. 1.

A network analyzer (Rohde \& Schwarz ZVR) is used to measure the complex channel frequency response for a set of transmitting and receiving antenna positions. The channel is probed in a $500 \mathrm{MHz}$ measurement bandwidth with a frequency resolution of $1.25 \mathrm{MHz}$ (401 frequency points) and a central frequency of $2.3 \mathrm{GHz}$. As transmitting (Tx) and receiving $(\mathrm{Rx})$ antenna, broadband omnidirectional biconical antennas of type Electro-Metrics EM-6116 are used. To be able to perform measurements for large Tx-Rx separations, one port of the network analyzer is connected to the Tx through an RF/optical link with an optical fiber of length $500 \mathrm{~m}$. A dirac pulse signal (in the frequency domain) generated by the network anlyzer is sent into the Tx via optical fiber. The RF signal sent into the Tx and the RF signal coming from the $\mathrm{Rx}$ are both amplified using an amplifier of type Nextec-RF NB00453 with an average gain of $37 \mathrm{~dB}$.

The amplifiers assure that the signal-to-noise ratio

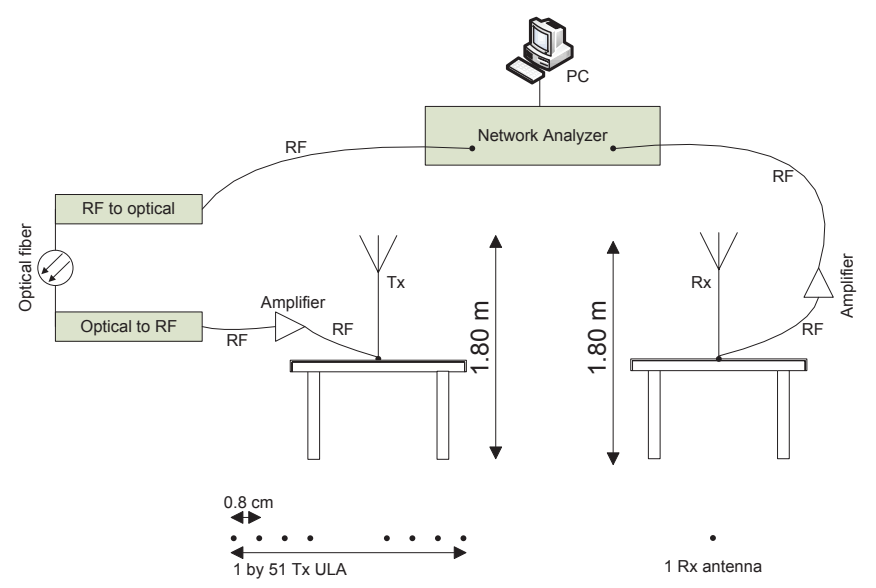

Fig. 1. Virtual channel sounder setup

at the receiving port of the network analyzer is at least $20 \mathrm{~dB}$ for each measured location of the Tx and Rx. The calibration of the network analyzer is done at the connectors of the Tx and Rx antenna, and as such includes both the RF/optical link and 
the amplifiers.

The Rx is fixed and the Tx is positioned in 51 different locations forming a virtual uniform linear array (ULA). Both antennas are polarized vertically and positioned at a height of $1.8 \mathrm{~m}$ during measurements. The separation between two adjacent positions in the array is $0.8 \mathrm{~cm}$. One measurement (all 51 virtual antenna positions of the array) last about 5 minutes. At each Tx position in the array, the network analyzer measures the $S_{21}$ scattering parameter ten times (i.e., 10 time observations), which we average to obtain one power delay profile (PDP) per Tx-Rx combination. Since we have $51 \mathrm{Tx}$ and $1 \mathrm{Rx}$, we have 510 PDPs for the whole ULA which is averaged to obtain the average PDP in $\mathrm{dB}$, labelled as APDP, and given by:

$$
\left.A P D P(t)\right|_{d B}=10 \log \left(\left|S_{21}^{a v}(t)\right|^{2}\right),
$$

where $S_{21}^{a v}(t)$ is the average PDP in linear domain over the number of Tx positions. The spatial averaging is necessary to avoid the effect of fading, it is performed by the stepper motor moving the antennas around. The PDP is plotted within a delay of $800 \mathrm{~ns}$, which is enough to observe the decay of the power. The scattering parameter $S_{21}$ is translated into the time domain by using an IFFT operation with an UNIFORM window (see Section III-A for motivations).

\section{B. Channel sounder setup}

The measurements were also carried out using a Multiple-Input Multiple-Output (MIMO) channel sounder, based on the correlation principle. The system allows truly simultaneous measurement of all up to $8 \mathrm{Rx}$ and $16 \mathrm{Tx}$ branches. Details of the measurement system can be found in [13]. The main characteristics of the setup used in this set of measurements are:

- Measurements time-triggered at $60 \mathrm{~Hz}$. This ensures proper sampling of the channel, which is changing due to both movements of the $\mathrm{Rx}$ and other external changes.

- Bandwidth: $100 \mathrm{MHz}$

- Carrier frequency: $2.3 \mathrm{GHz}$.

- Measurement duration: 10 seconds (600 measurements)

- Rx channels: 4 mockup laptop arrays are measured simultaneously, each with 4 antennas (measured via switch). The laptop arrays have ${ }^{3}$ 2 top-mounted and 2 side-mounted dipole-like antennas.

- Tx channels: 4 dual-band branches, 4 element linear array with vertically polarized elements. Each of the four laptops is placed on a table in front of a person. During each measurement the persons are moving the laptops randomly on the table with displacements of approximately plus $/$ minus $10 \mathrm{~cm}$. For the current work, the PDPs are obtained as an average over all the measurements made with the four antennas on the laptop.

\section{Description of the environment}

1) Environment in Aalborg (Denmark): The measurements were performed in a seminar room of Aalborg University (AAU), shown in Fig. 2, where only the right part is used. The room is equipped with tables, chairs, and ordinary meeting room facilities. The locations for $\mathrm{Rx}$ in this room are summarized

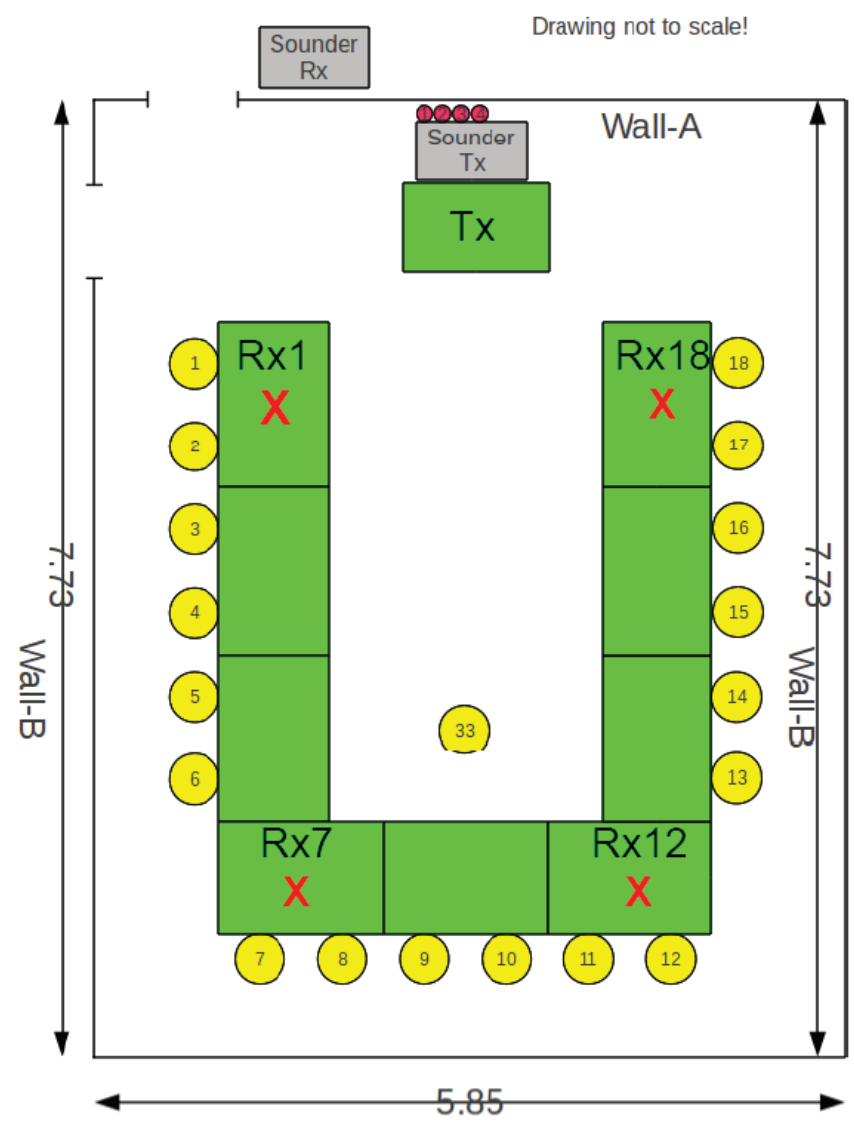

Fig. 2. Seminar room: Tx and Rx measurement positions 
in Table I.

The Tx had a fixed position whereas the Rx occupies positions $\mathrm{Rx} 1, \mathrm{R} \times 7, \mathrm{Rx} 12$, and $\mathrm{Rx} 18$ as shown in Fig. 2. The measurements with the channel sounder

\begin{tabular}{|l|l|l|}
\hline Position & $\begin{array}{l}\text { Distance to } \\
\text { Wall A (m) }\end{array}$ & $\begin{array}{l}\text { Distance to } \\
\text { Wall B (m) }\end{array}$ \\
\hline Rx1 & 2.5 & 4.6 \\
\hline Rx7 & 6.4 & 4.4 \\
\hline$R \times 12$ & 6.5 & 1.4 \\
\hline Rx18 & 2.5 & 1 \\
\hline
\end{tabular}

TABLE I

LOCATIONS FOR RX IN THE SEMINAR ROOM IN AALBORG

occured in May, 2010.

2) Environment in Ghent (Belgium): The measurements were performed in room $\mathrm{C} 3 / 3-3$ and $\mathrm{C} 3 / 3$ 4 at the third floor of an office building (Ghent, Belgium). The ground plan is shown in Fig. 3. This floor is comprised of different rooms indexed by $C 3 / 3-i$ where $i$ is the $i^{\text {th }}$ room. We are only interested in room C3/3-3 and C3/3-4. A description of these rooms is as follows: room $\mathrm{C} 3 / 3-3$ contains desks (with chairs, computers, etc...), people, dressers and other furniture such as cardboards, books, etc... and the room C3/3-4 contains about the same things. The receiver is always located in room

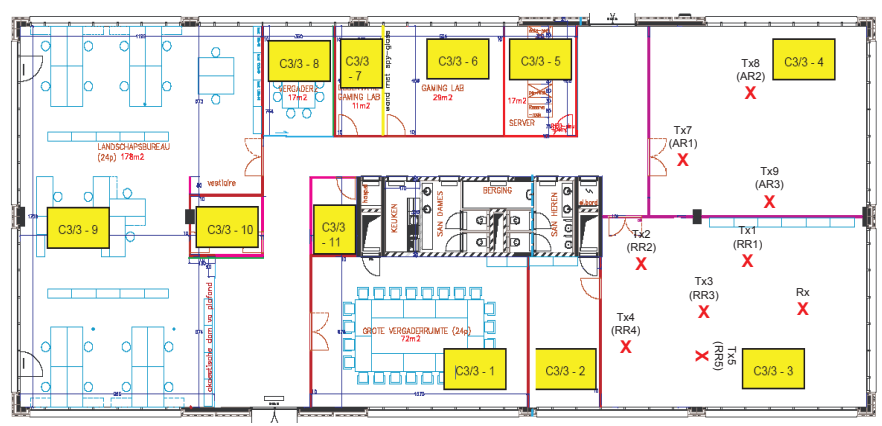

Fig. 3. Third floor of office building in Ghent

C3/3-3 whereas the transmitter is either located in room C3/3-3 (Room-Room, indexed by RR), or in room C3/3-4 (Adjacent-Room, indexed by AR). The measurements with the virtual channel sounder were performed from September $20^{\text {th }}$ to September $24^{\text {th }}$, 2010 .

\section{Methodology}

\section{A. Extraction of the reverberation time $(\tau)$}

In indoor propagation, multiple reflections and scattering give rise to a tail with an exponential decay in the PDP curve and a time constant noted as the reverberation time [7]. In a $\log$-scale this is equivalent to a tail of the impulse response which is linear, and its slope leads to the reverberation time according to the following formula:

$$
\tau=-\frac{10 \log (e)}{\text { slope }}
$$

where $e=2.718 \ldots$ is the Euler's number and slope is the slope of the linear tail in the impulse response. For the assessment of $\tau$, the amplitudes of the average PDP at different delays don't have to be accurate (because we are interested in the slope), instead the frequency resolution, i.e., the temporal resolution has to be as accurate as possible. This is the reason why the UNIFORM window has been used in the IFFT computation, since it has a NoisePower-Bandwidth factor of 1.

Actually the experimental APDP (in dB) does not have a perfect linear tail because of the noise level of the network analyser, the measurement uncertainties, etc. We need to derive the reverberation time over a certain delay range (or a certain range of power) for which the APDP tail is approximated by linear regression. This choice should be done automatically because the manual detection would suffer from judgement subjectivity (see next section). The methodology is shown in the flow graph of Fig. 4.

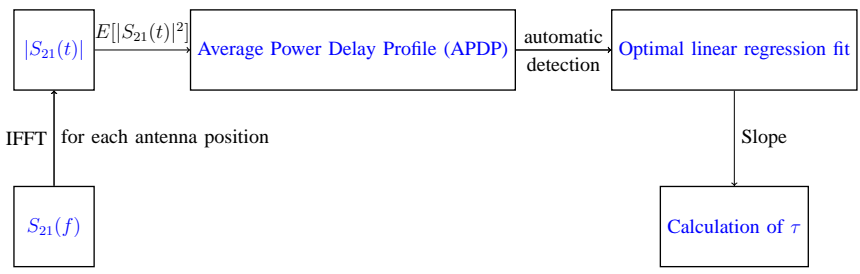

Fig. 4. Flow chart of methodology for virtual channel sounder method

\section{B. Automatic detection algorithm}

In this section we introduce the algorithm (in Fig. 5) used for the automatic reverberation time 
detection. One may detect automatically the reverberation time using a fixed delay range (or a fixed power range). Such kind of algorithm would be good for a certain room and propagation environment. But when changing the room (mainly the volume), the Tx-Rx separation for the same room, or when the propagation environment changes, the received power may be different. Then the PDP tail varies and neither the delay range, nor the power range will be valid. We define here different quantities using the

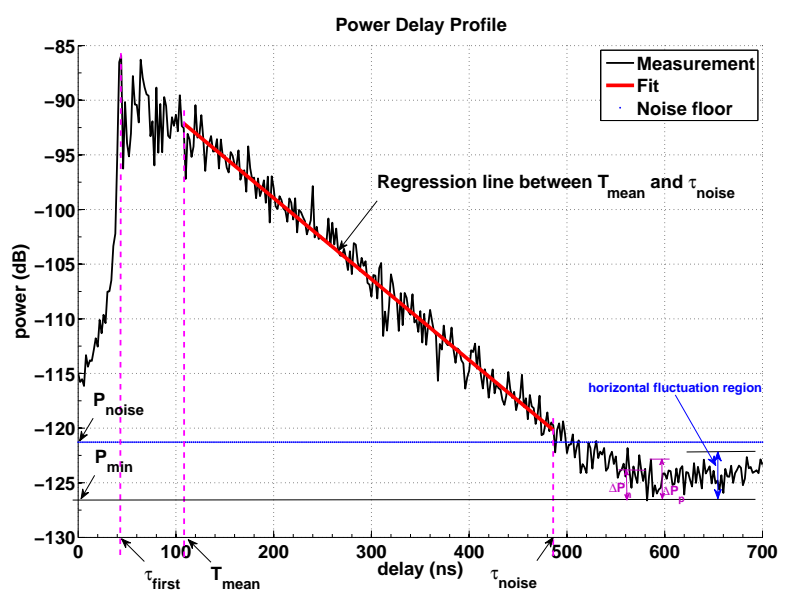

Fig. 5. Power Delay Profile (transceiver in adjacent room) and explanation of automatic detection algorithm

APDP curve (as in Fig. 5) to explain the algorithm:

- $\tau$ first: the arrival time of the first component,

- $T_{\text {mean }}$ : the mean arrival time,

- $P_{\text {min }}:$ the minimum APDP value,

- $\Delta P_{n}$ : the difference between $P_{\min }$ and the value of the first peak before $P_{\text {min }}$.

- $\Delta P_{p}$ : the difference between $P_{\min }$ and the value of the first peak after $P_{\min }$.

The following threshold is then defined:

$$
\text { Threshold }=P_{\text {min }}+\min \left(\Delta P_{n}, \Delta P_{p}\right),
$$

where $\min (a, b)$ is the minimum value of $a$ and $b$, we introduce $\Delta P_{n}$ and $\Delta P_{p}$ to ensure that the Threshold will be in the noise zone (see Fig. 5).

All APDP values lower than Threshold are gathered in an array, called "NoiseArray". We finally define the noise floor by:

$$
P_{\text {noise }}=\operatorname{mean}(\text { NoiseArray })+3 \mathrm{~dB},
$$

where $3 \mathrm{~dB}$ is added to be 2 times above the noise mean value.
It should be noted that the algorithm described in this section is only used for the ULA measurements.

Let's denote by $\tau_{\text {noise }}$ the delay corresponding to $P_{\text {noise }}$. The reverberation time is derived by performing a linear regression fit between $T_{\text {mean }}$ and $\tau_{\text {noise }}$ as shown in Fig. 5, with:

$$
T_{\text {mean }}=\frac{\int \tau P(\tau) d \tau}{\int P(\tau) d \tau},
$$

where we replace $\operatorname{APDP}(\tau)$ by $\mathrm{P}(\tau)$ for notation convenience.

\section{Absorption area and absorption cross section (ACS)}

When electromagnetic fields propagate in a room, rays impinge walls, objects, ceiling, possibly hu$\operatorname{man}(\mathrm{s})$, etc... and this results in an absorption area, i.e., the total surface absorbed by radiation. Considering all objects fixed (except people), the absorption area will only vary according to the number of people in the room. The total effective absoption area is given by:

$$
A_{n}^{\prime}=A_{0}^{\prime}+n \times A C S,
$$

where $A_{n}^{\prime}$ is the total effective absorbing area, $A_{0}$ is the effective absorbing area without people, $n \in \mathbb{N}$ is the number of people in the room, and $A C S$ is the absorption cross section (we assume that all present persons have the same ACS), i.e., the human body surface absorbing electromagnetic radiation.

The methodology is based on Room electromagnetics theory [7], which states that all losses in a closed room can be described with a single parameter, called the reverberation time and is determined from the PDP (see section. III-A).

From the reverberation time [7] it is possible to assess the effective absorption area $\left(A_{n}^{\prime}\right)$ :

$$
A_{n}^{\prime}=\frac{4 V}{c \tau_{n}}
$$

where $V, \mathrm{c}=3 \times 10^{8} \mathrm{~m} / \mathrm{s}$ and $\tau_{n}$ are the room volume, the light velocity and the reverberation time when $n$ person(s) are present in the room, respectively.

By varying the room occupation with people, the effective absorbing areas as a function of the number of people $(n=0,2,3,7,10$, etc...) is obtained from the reverberation times values. The absorption cross section is then the slope of the linear regression of 
the points related to the different absorbing areas for the different number of people (see Section V-A).

\section{Calculation of SAR from ACS and vice-versa}

As stated in Section I, the SAR is the physical quantity used for defining the basic restrictions [8]. Basically, the $\mathrm{SAR}_{w b}$ is defined as the amount of power absorbed by an individual per mass unity, and is given by:

$$
\begin{aligned}
S A R_{w b} & =\frac{P_{a b s, \text { pers }}}{m} \\
& =\frac{P_{a b s, \text { pers }, \text { dif }}+P_{a b s, \text { pers }, \text { los }}}{m},
\end{aligned}
$$

where $P_{a b s, p e r s}, P_{a b s, p e r s, d i f}, P_{a b s, p e r s, l o s}$ are the total power absorbed, the diffuse power absorbed, the power absorbed due to LOS component respectively by a person in $\mathrm{W}$ and $\mathrm{m}$ is his weight in $\mathrm{kg}$.

In room electromagnetics analysis, a person is exposed to two sources of power, i.e., the diffuse scattered field from the reverberation phenomenon and the coherent power from the LOS component. Firstly, the power absorbed due to the diffuse multipath components (DMC) is determined. The power density in diffuse field has been investigated in [1], and expressed as follows:

$$
I_{d i f}=\frac{1}{\pi A^{\prime}} e^{-\frac{d_{0}}{c \tau}} * \eta_{p o l},
$$

where $d_{0}, \lambda$ and $\eta_{\text {pol }}$ are the shortest distance (separation) between the transceivers, the wavelength and the polarization factor of the DMC, respectively. Assume that the DMC is completely diffuse (the polarization is then completely random), a polarization factor of 0.5 is considered.

The power absorbed by a person in a diffuse field is defined as:

$P_{a b s, p e r s, d i f}=\int_{0}^{2 \pi} \int_{0}^{\pi} I_{d i f}^{\prime}(\theta, \phi) A(\theta, \phi) \cos \theta \sin \theta d \theta d \phi_{t}$

where $I_{d i f}^{\prime}(\theta, \phi)$ is the receiving radiance in $W . s r^{-1} \cdot m^{-2}$. Because in completely diffuse field the intensity is independent on the direction (azimut- elevation), Eq. 9 is now expressed as:

$$
\begin{aligned}
P_{a b s, p e r s, d i f} & =I_{d i f}^{\prime} \int_{0}^{2 \pi} \int_{0}^{\pi} A(\theta, \phi) \cos \theta \sin \theta d \theta d \phi \\
& =I_{d i f} \times A_{\text {average }} \\
& \equiv I_{d i f} \times A C S
\end{aligned}
$$

where $I_{d i f}, A(\theta, \phi)$ are the incoming irradiance in $W . m^{-2}$ and the absorption cross section from an incident plane wave, respectively.

In general, $A(\theta, \phi)$ is not known excepted from numerical calculations. From the measurements, we know the average whole-body absorption cross section $\left(A_{\text {average }}\right)$, which is equivalent to the $A C S$.

Secondly the power absorbed by a person due to the LOS component is:

$$
\begin{aligned}
P_{a b s, p e r s, l o s} & =I_{l o s} \times A\left(\theta_{0}, \phi_{0}\right) \\
& =I_{l o s} \times A_{l o s}
\end{aligned}
$$

where $I_{l o s}$ is the LOS component power density, which is direction-independent because an antenna with isotropic radiation pattern (fields coming from all directions with the same magnitude) is used.

The power density for the LOS component $\left(I_{l o s}\right)$ is computed from the received LOS component and the receiving area of the $\mathrm{Rx}$ antenna and will be investigated in Section V-B.

$A\left(\theta_{0}, \phi_{0}\right)$ is still unknown, but let assume the following: the LOS component only sees one side of the body, and the front and back of a body are identical in term of surface. So we can say that $A_{\text {los }}=\frac{A_{\text {average }}}{2}=\frac{A C S}{2}$, hence Eq. 11 becomes:

$$
P_{a b s, p e r s, l o s}=I_{l o s} \times \frac{A C S}{2}
$$

Using Eq. (7), Eq. (10) and Eq. (12) the whole-body SAR is defined as:

$$
S A R_{w b}=\frac{A C S\left(I_{t o t a l}+I_{d i f}\right)}{2 m},
$$

where $I_{\text {total }}=I_{\text {los }}+I_{\text {dif }}$ is the total incident power density.

\section{BENCHMARKING}

\section{. Channel sounder vs Virtual channel sounder}

In order to validate the measurement system, we compare the results of the channel sounder with those of the virtual channel sounder. The measurements campaign has been performed in the same 
room in Aalborg with both measurement systems. The room volume is about $118 \mathrm{~m}^{3}$. The measurements have been done at different dates and the Tx and Rx locations were the same (as best as possible) for the two measurement campaigns (see Section II). Tables II and III summarize the results for the reverberation time obtained for both measurement systems. D, Pers., $T_{\text {mean }}$, and $A^{\prime}$ represent the Tx$\mathrm{Rx}$ separation, the number of persons in the room, the mean-delay arrival time of the rays and the absorption area respectively.

As we are mainly interested in the reverberation

\begin{tabular}{|l|l|l|l|l|l|}
\hline Position & $\begin{array}{l}\mathrm{D} \\
(\mathrm{m})\end{array}$ & Pers. & $\begin{array}{l}\tau \\
(\mathrm{ns})\end{array}$ & $\begin{array}{l}T_{\text {mean }} \\
(\mathrm{ns})\end{array}$ & $\begin{array}{l}A^{\prime} \\
\left(\mathrm{m}^{2}\right)\end{array}$ \\
\hline Rx1 & 3.4 & 6 & 23.1 & 15.9 & 68 \\
\hline Rx7 & 4 & 6 & 22.7 & 18.2 & 69.3 \\
\hline Rx12 & 6.2 & 6 & 26.8 & 19.6 & 61.2 \\
\hline Rx12 & 6.2 & 9 & 25.5 & 19.7 & 61.7 \\
\hline Rx12 & 6.2 & 12 & 25.3 & 20 & 62.1 \\
\hline Rx12 & 6.2 & 15 & 26.1 & 21 & 60.3 \\
\hline Rx18 & 2.7 & 12 & 25.3 & 20 & 62.1 \\
\hline
\end{tabular}

TABLE II

PARAMETERS FROM CHANNEL SOUNDER MEASUREMENT

\begin{tabular}{|l|l|l|l|l|l|}
\hline Position & $\begin{array}{l}\mathrm{D} \\
(\mathrm{m})\end{array}$ & Pers. & $\begin{array}{l}\tau \\
(\mathrm{ns})\end{array}$ & $\begin{array}{l}T_{\text {mean }} \\
(\mathrm{ns})\end{array}$ & $\begin{array}{l}A^{\prime} \\
\left(\mathrm{m}^{2}\right)\end{array}$ \\
\hline Rx1 & 3.6 & 0 & 24.5 & 26.2 & 64.2 \\
\hline Rx1 & 3.6 & 2 & 23.8 & 26.2 & 66.2 \\
\hline Rx7 & 7.2 & 2 & 25.2 & 42.1 & 62.5 \\
\hline Rx7 & 7.2 & 4 & 25.1 & 40 & 62.7 \\
\hline Rx7 & 7.2 & 18 & 22 & 38.5 & 71.5 \\
\hline Rx12 & 6 & 2 & 28.2 & 39.5 & 55.8 \\
\hline Rx18 & 3 & 2 & 26 & 16.5 & 60.6 \\
\hline
\end{tabular}

TABLE III

PARAMETERS FROM VIRTUAL CHANNEL SOUNDER MEASUREMENT

time $\tau$, we focus the comparison on this parameter as illustrated in the diagram chart in Fig. 6. For the channel sounder we obtain a mean value of $\tau$ $\left(\tau_{\text {mean }}\right)$ equal to $24.5 \mathrm{~ns}$ with a standard deviation $\sigma_{\tau}$ equal to $1.7 \mathrm{~ns}$ which is $6.9 \%$ of $\tau_{\text {mean }}$. We obtain $\tau_{\text {mean }}=25.8 \mathrm{~ns}$ and $\sigma_{\tau}=1.6 \mathrm{~ns}(6.2 \%$ of $\left.\tau_{\text {mean }}\right)$ for the virtual channel sounder. The deviation between reverberation time obtained with the two measurement systems is only about $1.3 \mathrm{~ns}$ (5.2\% of

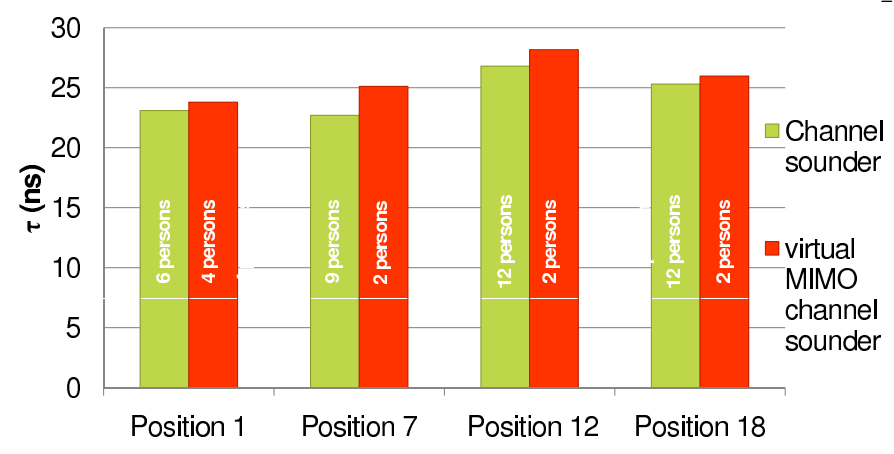

Fig. 6. Channel sounder and the virtual channel sounder

their mean value).

The observed small discrepancies between the results of the two measurement systems may be due to the following reasons:

- differences between the measurement system setups

- variations in Tx-Rx positions: for a certain position, the Tx-Rx separation may not be exactly the same for the two measurement campaigns (second column of Tables II and III). The theory actually says that the reverberation time should be independent on the location, assuming only diffuse scattering.

- furniture, tables, people, etc ... were not exactly in the same positions for both measurement campaigns

- different number of people in the room as illustrated in the diagram chart (Fig. 6)

Given the small deviations between the reverberation time obtained for each measurement system at different locations, we can say that the reverberation time is independent of the transceiver location, as suggested by the room electromagnetics theory [7], regardless of the measurement system. However, we observe that the results obtained with the channel sounder are systematically lower than the ones obtained with the virtual channel sounder, this might be due to different polarization of the used antennas.

\section{B. Different antennas}

Using the virtual channel setup, we investigated the reverberation time variation using different antennas. We first use a broadband omnidirectional 
biconical antenna of type Electro-Metrics EM- 6116 denoted "ibbt" and the "laptop antennas" mentioned in Section II-B denoted here as "aau". For the

\begin{tabular}{|l|l|l|l|l|l|l|}
\hline Position & $\begin{array}{l}\mathrm{D}_{\text {ibbt }} \\
(\mathrm{m})\end{array}$ & $\begin{array}{l}\mathrm{D}_{\text {aau }} \\
(\mathrm{m})\end{array}$ & $\begin{array}{l}\text { Pers. } \\
(\mathrm{ibbt})\end{array}$ & $\begin{array}{l}\text { Pers. } \\
(\mathrm{aau})\end{array}$ & $\begin{array}{l}\tau_{(i b b t)} \\
(\mathrm{ns})\end{array}$ & $\begin{array}{l}\tau_{(\text {aau })} \\
(\mathrm{ns})\end{array}$ \\
\hline Rx1 & 3.6 & 3.6 & 1 & 1 & 24.1 & 24.9 \\
\hline Rx7 & 7.2 & 6.6 & 2 & 1 & 25.2 & 23.7 \\
\hline Rx12 & 6 & 6 & 2 & 1 & 28.2 & 25.8 \\
\hline Rx18 & 3 & 2.4 & 2 & 1 & 26 & 22 \\
\hline
\end{tabular}

TABLE IV

PARAMETERS FOR DIFFERENT ANTENNAS USING THE VIRTUAL CHANNEL SOUNDER, IBBT $=$ BICONICAL ANTENNA AND AAU $=$ LAPTOP ANTENNA

biconical antenna (ibbt) we obtain a mean value of $\tau\left(\tau_{\text {mean }}\right)$ about $25.9 \mathrm{~ns}$ whereas we obtain $\tau_{\text {mean }}$ $=24.1 \mathrm{~ns}$ for the laptop antenna, which gives a difference of $1.8 \mathrm{~ns}$ (7.2\% of their mean value). Table IV summarize the results for different antennas. Few variations between the results are observed, but this was expected due the reasons mentioned in Section IV-A except that we used here the same measurement system. Again we find almost the same values of the reverberation time using one measurement system with different antennas, indicating that the reverberation time is independent of the antennas although it should be noted that the polarization might have a slight influence if a special algorithm is not used to extract only the diffuse part.

\section{Reverberation time in adjacent room}

Up to now, reberberation times were investigated for Tx-Rx located in the same room (see Section IV-A).

The reverberation time when the transmitter (Tx) is located in a room adjacent of the receiver $(\mathrm{Rx})$ room is investigated here. The transmitter is located in room $\mathrm{C} 3 / 3-4$ and the receiver is located in room C3/3-3 (see Fig. 3). The basic theory is the same as the case where the transmitter is located in the same room, unless that there is an energy exchange between the two rooms for the coupling elements case [6]. Let the transmitter be located in the room $R_{T x}$ (C3/3-4) and the receiver in the room $R_{R x}$ (C3/3-3). Both rooms are closed and seperated by a wall with surface $S . \mathrm{P}(\mathrm{t})$ is the power transmitted in room $R_{T x}, \alpha$ is the wall transmission factor.

In the following we assume complete diffuse field in both rooms, then the power density is directionindependent, i.e., $I(\theta, \varphi)=I$.

The room $R_{R x}$ is fed by two sources:

- $\alpha^{2} P(t)$, which is the transmitted LOS power scaled by the wall transmission factor

- $P_{\text {exch }}$, which is due to the exchanged energy from $R_{T x}$ to $R_{R x}$ through surface $S$ by the diffuse field.

These received input powers in room $R_{R x}$ are balanced by the increase in energy per second and the losses through walls as follows [6]:

$$
\alpha^{2} P(t)+P_{\text {exch }}=V \frac{d W_{R x}}{d t}+\frac{c \eta A}{4} W_{R x}
$$

where $V$ is the volume of the room where the Rx is located, $\eta$ is the wall absorption factor, and $A$ is the total absorbed area.

The energy $W$ and the power density $I$ in the two rooms are related as follows: $W_{R x}=\alpha^{2} \times W_{T x}$ and $I_{R x}=\alpha^{2} \times I_{T x}$. Assuming that the radiance $I_{T x}(\theta, \varphi)$ is in the diffuse field and impinges uniformly on wall $S$ (with $\alpha$ being its transmission factor), then the transmitted power via the surface $S$ to the room $R_{R x}$ is:

$$
\begin{aligned}
P_{\text {exch }} & =S \int_{0}^{2 \pi} \int_{0}^{\frac{\pi}{2}} \alpha^{2} I_{T x}(\theta, \varphi) \sin (\theta) \cos (\theta) d \theta d \varphi \\
& =\pi \alpha^{2} I_{T x} S \\
& =\pi I_{R x} S
\end{aligned}
$$

Using $W_{R x}=4 \pi \frac{I_{R x}}{c}$ [7] ( $c$ is the light velocity in the vacuum), $P_{\text {exch }}$ is expressed as a function of $W_{R x}$ as follows:

$$
P_{\text {exch }}=\frac{c S}{4} W_{R x},
$$

If the source is turned off i.e., $P(t)=0$ and using Eq. (15), Eq. (14) becomes:

$$
V \frac{d W_{R x}}{d t}+\frac{c}{4}(\eta A-S) W_{R x}=0
$$

which is a homogeneous equation with the solution

$$
W_{R x}=W_{0} e^{-\frac{t}{\tau}},
$$

with

$$
\tau=\frac{\tau_{0}}{1-\frac{S}{\eta A}},
$$


$\tau_{0}=\frac{4 V}{c \eta A}$ is the reverberation time when both $\mathrm{Tx}$ and $\mathrm{Rx}$ are located in the same room $R_{R x}$ i.e., room $\mathrm{C} 3 / 3-3, \eta A=A^{\prime}$ is the effective area of the total absorbing surface in room $R_{R x}$ and $\mathrm{V}$ is the volume of the room $R_{R x}$. The volume $\mathrm{V}$ is roughly about $360 \mathrm{~m}^{3}$.

From Eq. (16), it is easy to check that $\tau>\tau_{0}$ for $S<A^{\prime}$. Physically, $\tau>\tau_{0}$ could be explained by the exchanged energy between both room, making the reverberation time more longer. The measurement

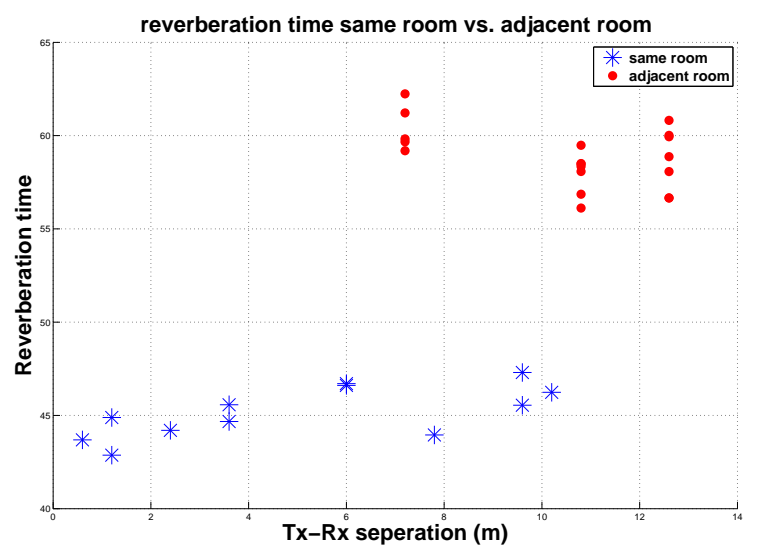

Fig. 7. Reverberation time investigation

results are shown in Fig. 7. The mean value of the reverberation time when the transceivers are located in the same room and adjacent room are $\tau_{0}=45.32 \mathrm{~ns}$ and $\tau=58.90 \mathrm{~ns}$, respectively. It is clear from Fig. 7 that $\tau_{0}$ (Tx and $\mathrm{Rx}$ in the same room) is lower than $\tau$ (Tx and Rx in adjacent room) as shown in Eq. (16). The variation of the reverberation time in both cases is related to the number of people in the room, given that this influences the total absorbing area and hence the reverberation time.

From the measurements in Fig. 7 we obtain $\left.\frac{\tau_{0}}{\tau}\right|_{\text {meas }}=\frac{45.32}{58.90}=0.769$. From Eq. (16) we deduce $\left.\frac{\tau}{\tau}\right|_{\text {calc }}=1-\frac{S}{A^{\prime}}$. The surface $S$ being about $28 \mathrm{~m}^{2}$ and the effective area $A^{\prime}=105.7 \mathrm{~m}^{2}$ (derived from $\tau_{0}$ measurement), we obtain $\left.\frac{\tau_{0}}{\tau}\right|_{\text {calc }}=1-\frac{28}{105.7}=0.735$. This shows that the measurement results are in good agreement with the calculation of the theory as both ratios agree excellently.

\section{EXPERIMENTAL DETERMINATION OF THE $S A R_{w b}$}

\section{A. Absorption cross section}

The measurements were performed in an office environment (room C3/3-3, volume $\approx 360 \mathrm{~m}^{3}$ ) in Ghent, Belgium (see Fig. 3). Three spatial locations for the Tx and one location for the Rx are selected. Data of both frequencies $2.30 \mathrm{GHz}$ and $3 \mathrm{GHz}$ were used. An illustration of the absorption area as a function of room occupation is shown in Fig. 8. The measurement results in terms of absorption cross section for the different locations and the two frequencies are summarized in Table V. The average

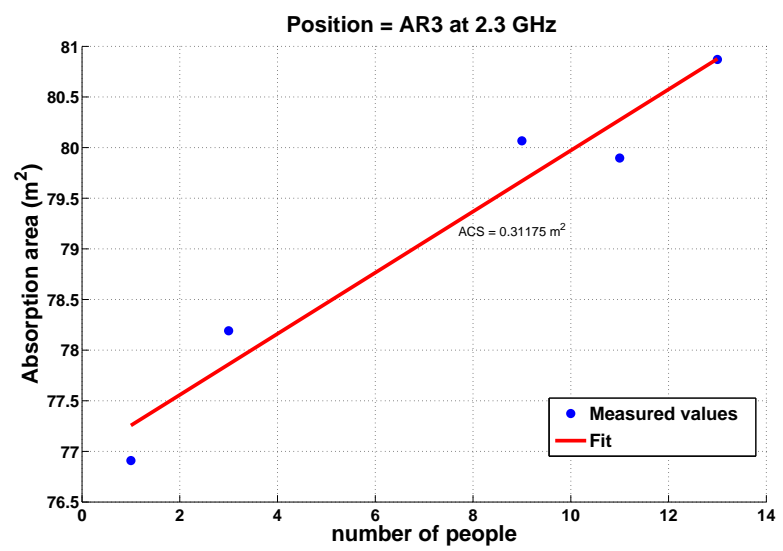

Fig. 8. Absorption area as a function of room occupation

\begin{tabular}{|l|l|l|}
\hline \multicolumn{2}{|c|}{ ACS $\left(\mathrm{m}^{2}\right)$} & \multirow{2}{*}{ Positions } \\
\cline { 1 - 2 } $\mathrm{f}=2.3 \mathrm{GHz}$ & $\mathrm{f}=3 \mathrm{GHz}$ & \\
\hline 0.42 & 0.24 & Position 1 (AR1) \\
\hline 0.28 & 0.43 & Position 2 (AR2) \\
\hline 0.31 & 0.42 & Position 3 (AR3) \\
\hline \hline 0.34 & 0.36 & $\begin{array}{l}\text { averaged over Position } \\
1,2 \text { and 3 }\end{array}$ \\
\hline
\end{tabular}

TABLE V

ABSORPTION CROSS SECTION (ACS) FROM MEASUREMENT DATA

ACS at $2.3 \mathrm{GHz}$ is about $\mathbf{0 . 3 4} \mathbf{~ m}^{2}$ and $\mathbf{0 . 3 6} \mathbf{~ m}^{2}$ at $3 \mathrm{GHz}$. We notice that the absorption cross section varies slightly as a function of the frequency.

Assuming that the mean weight and height of people under test are $70 \mathrm{~kg}$ and $175 \mathrm{~cm}$ respectively, we obtain from Fujimoto's formula [14] a mean body surface area (BSA) of about $1.79 \mathrm{~m}^{2}$. From the 
correlation between the BSA and the ACS at $2 \mathrm{GHz}$ [12], we derive an ACS of about $0.49 \mathrm{~m}^{2}$. Recently, [15] investigated the surface of the Visual Human $(\mathrm{VH})$ illuminated by a plane wave at $2.1 \mathrm{GHz}$ for three orientations (front, right side and oblique). An illuminated surface of about $0.44 \mathrm{~m}^{2}, 0.23 \mathrm{~m}^{2}$ and $0.39 \mathrm{~m}^{2}$ has been found for the front, right side and oblique orientation, respectively.

It should be acknowledged that the persons may not be exposed identically, leading to the observed small differences.

\section{B. Power density}

As already stated in Section III-D, power illuminating a person is absorbed in two ways in a closed environment, namely by the LOS component and by the diffuse part. There may be also a power absorption due to the specular components (caused by specular reflections), but we do not take this into account in this paper because of the room electromagnetics theory simplification. In this section we investigate the power density of these two components for the transceivers located in the same room. The LOS component power density is calculated from the experimental received power at a certain position and the Rx receiving area. The LOS component power density is, [16]:

$$
P_{d}=\frac{P_{r}}{A_{\text {eff }}}
$$

where $P_{d}, P_{r}$ and $A_{\text {eff }}$ are the power density, the received power, and the effective area of the receiving antenna.

The use of the hypothetical isotropic antenna allows to write that $A_{\text {eff }}=A_{\text {isotr. }}=\frac{\lambda^{2}}{4 \pi}$, [16]. Since we measure the received power, and the wavelength is known, it is then easy to derive the power density from Eq. (17). Its expression is as follows:

$$
P_{d}=4 \pi \frac{P_{r}}{\lambda^{2}}
$$

This is the experimental way to measure the power density of the LOS component. Since the power density of the LOS component is determined from the PDP, the LOS component amplitude is of main importance whereas its arrival time is non-relevant. The FLAT TOP window has the best amplitude accuracy because of its largest Wide-Main-Lobe.
The FLAT TOP window has been used in the IFFT computation leading to the determination of the LOS component power density.

Theoretically from a distance $d$ of the transmitter it is expressed as:

$$
P_{d}=\frac{P_{t}}{4 \pi d^{2}}
$$

where $P_{t}$ is the radiated power by the transmitting antenna.

The diffuse power density is derived at a certain position using Eq. (8) once the reverberation time and the absorption area are known. Fig. 9 shows the power densities (LOS, diffuse) for different distances in the same room (room $\mathrm{C} 3 / 3-3$ in Fig. 3) for an input power of $1 \mathrm{~W}$. The experimental LOS power density agrees with the theoretical LOS power density.

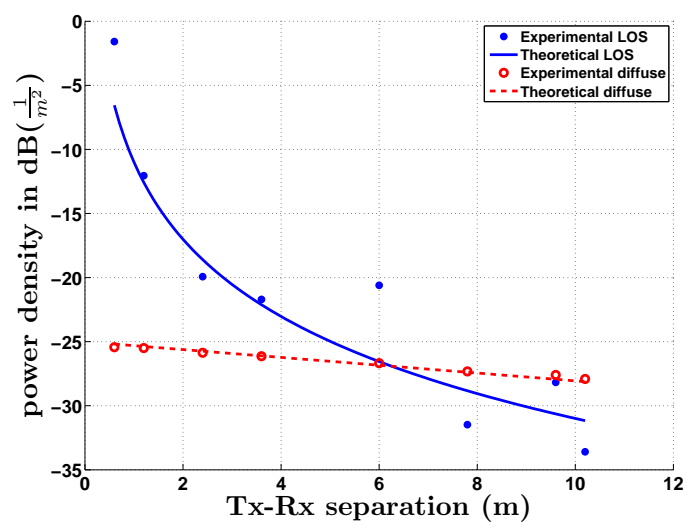

Fig. 9. Power density for $1 \mathrm{~W}$ input as a function of separation between $\mathrm{Tx}$ and $\mathrm{Rx}$

The LOS power absorption is important in the vicinity of the tranmitting antennas, i.e., in a radius smaller than $4 \mathrm{~m}$. As we move away from the transmitter, the LOS power density decreases dramatically, making the power absorption less significant for higher distances. Experimental and theoretical results for the diffuse power density agree very well, although it should be admitted that the theoretical results use the experimental reverberation time and absorption area (only the distance is actually theoretical), see Eq. (8).

The diffuse power also decreases with increasing separation. As shown in Fig. 9 the LOS field dominates the diffuse field in the vicinity of the transmitter, whereas the diffuse field is dominating for 
long Tx-Rx separation. This leads to the so-called reverberation distance. At the reverberation distance location, the LOS field and the diffuse field have the same intensity. Its theoretical expression has been investigated in [7], and is defined as:

$$
R_{d}=\frac{1}{2} \sqrt{D_{t} D_{r} A^{\prime}}
$$

where $R_{d}, D_{t}, D_{r}$ are the reverberation distance, the transmitter and the receiver antenna directivity, respectively.

In a completely diffuse field, the power density is direction-independent, thus the directivity of the antennas is unity, i.e., $D_{t}=D_{r}=1$. The results of the reverberation distance according to the Tx-Rx position are shown in Table VI. We obtain a mean

\begin{tabular}{|l|l|l|l|l|l|l|l|l|}
\hline $\begin{array}{l}\text { Tx-Rx sep- } \\
\text { aration (m) }\end{array}$ & 0.6 & 1.2 & 2.4 & 3.6 & 6 & 7.8 & 9.6 & 10.2 \\
\hline$R_{d}(\mathrm{~m})$ & 5.2 & 5.3 & 5.2 & 5.2 & 5.1 & 5.2 & 5.0 & 5.1 \\
\hline
\end{tabular}

TABLE VI

REVERBERATION DISTANCES

reverberation distance of about $5.16 \mathrm{~m}$ while a standard deviation of about $0.09 \mathrm{~m}$ is obtained. This $R_{d}$ agrees excellently with the intersection of the LOS and diffuse power density in Fig. 9. So we conclude that the measurement is in good agreement with the theory.

The slope of the decrease of the diffuse power derived from Eq. (8) is $-\frac{10 \times \log _{10}(e)}{c \tau}$ (in $\mathrm{dB}$ ), which is only dependent on the reverberation time, meaning that in a large room the scattered power density tends to be constant, or at least within a certain range of Tx-Rx separation. For the room investigated here $(\tau \approx 45 \mathrm{~ns}$ ), the slope is about $0.32 \mathrm{~dB} / \mathrm{m}$ and hence we can consider the scattered power density as constant-we take the mean value-given that a maximal deviation between the extrema is only about $2 \mathrm{~dB}$.

\section{C. $S A R_{w b}$ calculation and application}

In this section the whole-body SAR is calculated using the method in Section III-D. The results will be compared with the absorption calculated using a statistical method [9], and with a calculation proposed in [10]. An absorption cross section of about $0.34 \mathrm{~m}^{2}$ (height $=175 \mathrm{~cm}$ and weight $=$ $70 \mathrm{~kg}$ ) at $2.3 \mathrm{GHz}$ has been obtained in Section $\mathrm{V}$-A. If we assume a total incident power density of $1 \mathrm{~W} / \mathrm{m}^{2}$, and a mean diffuse power density of about $2.3 \mathrm{~mW} / \mathrm{m}^{2}$ (see Section V-B), a whole-body SAR of $2.43 \mathrm{~mW} / \mathrm{kg}$ is obtained using the method proposed in Section III-D.

In [10] the whole-body averaged SAR calculation has been performed from $100 \mathrm{MHz}$ to $3 \mathrm{GHz}$ at the basic $2 \mathrm{~mm}$ resolution of the voxel model NORMAN using the Finite-difference time domain (FDTD) algorithm. An $\mathrm{SAR}_{w b}$ of about $3.77 \mathrm{~mW} / \mathrm{kg}$ has been found at $3 \mathrm{GHz}$. It is noteworthy to mention that the NORMAN phantom characteristics used in [10] are different from the characterictics used in the present paper. In [9] a statistical method is applied for the $\mathrm{SAR}_{w b}$ calculation in an indoor pico-cell environment at $2.45 \mathrm{GHz}$ with a spheroid average man with same characteristics (weight and height) as for our experiment and a mean $\mathrm{SAR}_{w b}$ of about $2.7 \mathrm{~mW} / \mathrm{kg}$ has been obtained. We obtain excellent agreement between this result and our measured $\mathrm{SAR}_{w b}$. [17] investigated the whole-body SAR for different phantoms from $20 \mathrm{MHz}$ to $2.4 \mathrm{GHz}$. A whole-body specific absorption rate of about $3.75 \mathrm{~mW} / \mathrm{kg}$ is found for the Visual Human, Zubal and Korean model at $2.4 \mathrm{GHz}$. For the Norman model a whole-body specific absorption rate of $6.25 \mathrm{~mW} / \mathrm{kg}$ is found at $2.4 \mathrm{GHz}$ whereas a value of $7 \mathrm{~mW} / \mathrm{kg}$ is found for the female Japan and Japan model at the same frequency. This paper highlights the variability of the whole-body averaged SAR according to the phantoms.

The small deviation from our experimental $\mathrm{SAR}_{w b}$ and the results of [9], [10], [17] may be explained by the difference in the phantom shapes and the postures. For instance, for the same body surface area of $1.78 \mathrm{~m}^{2}$ the absorption cross section variation may reach $0.15 \mathrm{~m}^{2}$ for two different phantoms shapes and/or postures [12], resulting a difference of $1.07 \mathrm{~mW} / \mathrm{kg}$ in the $\mathrm{SAR}_{w b}$. Compared to the results obtained in [9], [10], [17], mention that our method leads to the lowest value in term of absorption. This is mainly due to the fact that we have determined the absorption cross section in an office room with people working, meaning all the people in the room were sitting whereas phantoms 
are standing in the other papers.

As an application we investigate now the $\mathrm{SAR}_{w b}$ using an actual transmitted power for WiFi to show that our method enables to estimate whole-body SAR without the need of simulations. We assume an office scenario with a WiFi access point installed in a room. The maximum allowed EIRP (Effective

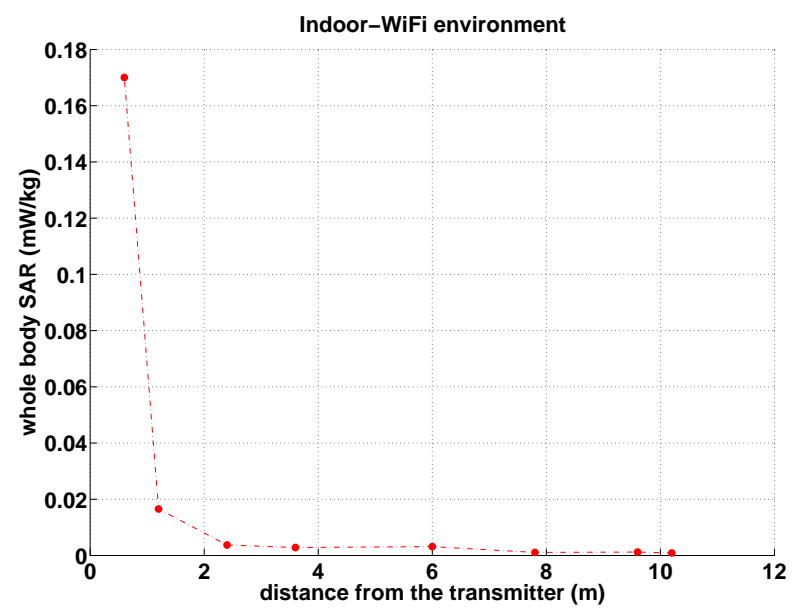

Fig. 10. Whole body SAR at $2.30 \mathrm{GHz}$ for $100 \mathrm{~mW}$ input as a function of distance (in the same room)

Isotropic Radiated Power) for 802.11 wireless LAN in $2.4 \mathrm{GHz}$ band is $100 \mathrm{~mW}$ [18]. Using this transmitted power and the power density of Section V-B, the $\mathrm{SAR}_{w b}$ can be determined. Fig. 10 shows $\mathrm{SAR}_{w b}$ in a person for different distances from the access point radiating with an EIRP of $100 \mathrm{~mW}$. The $\mathrm{SAR}_{w b}$ in a person decreases of course with distance with values of $0.17 \mathrm{~mW} / \mathrm{kg}$ at $0.6 \mathrm{~m}$ and $0.0012 \mathrm{~mW} / \mathrm{kg}$ at $10 \mathrm{~m}$. These values are much lower than $0.08 \mathrm{~W} / \mathrm{kg}$, which is the limitation for the exposure of the public [8].

\section{CONCLusions}

Based on a simple theory called room electromagnetics, we have found interesting properties of the reverberation time. The difference in values measured by two systems and different antennas has been discussed. Results confirming that the reverberation time is location and antenna independent have been presented, although the antenna polarization might have a small influence. It is possible to make use of a virtual channel sounder to measure the reverberation time. Room electromagnetics theory has been applied for the first time in adjacent rooms, and the measurement results are in excellent agreement with the developed theory. Further, a novel and easy method to assess the absorption cross section has been proposed. We found an absorption cross section of about $0.34 \mathrm{~m}^{2}$ at $2.3 \mathrm{GHz}$ and $0.36 \mathrm{~m}^{2}$ at 3 $\mathrm{GHz}$, confirming that the measurement results are in good agreement with literature. The theory for the power absorption for the line of sight component and for the diffuse field has been investigated. Using the measurement-based absorption cross section, a whole-body SAR of $2.43 \mathrm{~mW} / \mathrm{kg}$ is found in a realistic environment, in agreement with computational and statistical methods. The main advantage of the proposed method is that it does not require any simulation nor phantom modeling. The method developed here holds only for the vertical polarization, our future research will investigate the correlation between the absorptions induced by a vertical and horizontal E fields. Hence, the present theory can be extended to the horizontal polarization case.

\section{ACKNOWLEDGMENT}

The research leading to these results has received funding from the European Union's Seventh Framework Programme ([FP7/2007-2013]) under grant agreement no 244149. The research is also partly funded by the Fund for Scientific Research - Flanders (FWO-V, Belgium) project G.0325.11N.

Wout Joseph is a Post-Doctoral Fellow of the FWOV (Research Foundation - Flanders). 


\section{LIST OF FIGURES}

Virtual channel sounder setup

Seminar room: Tx and Rx measurement positions

Third floor of office building in Ghent Flow chart of methodology for virtual channel sounder method . . . . . . . . Power Delay Profile (transceiver in adjacent room) and explanation of automatic detection algorithm . . . . . . . 5 Channel sounder and the virtual channel sounder. . . . . . . . . . . . 7 Reverberation time investigation ...

8 Absorption area as a function of room occupation ............. 9

9 Power density for $1 \mathrm{~W}$ input as a function of separation between $\mathrm{Tx}$ and $\mathrm{Rx}$. 10

10 Whole body SAR at $2.30 \mathrm{GHz}$ for 100 $\mathrm{mW}$ input as a function of distance (in the same room) .........
LIST OF TABLES

2 I Locations for $\mathrm{Rx}$ in the seminar room in Aalborg . . . . . . . . . . . . 4

3 II Parameters from channel sounder mea-

4 surement . . . . . . . . . . 7

III Parameters from virtual channel 4 sounder measurement . . . . . . . . 7

IV Parameters for different antennas using the virtual channel sounder, ibbt $=$ biconical antenna and aau = laptop antenna 8

$\mathrm{V}$ Absorption cross section (ACS) from measurement data . . . . . . . . . 9 9

9 VI Reverberation distances ........ 11 


\section{REFERENCES}

[1] Jørgen Bach Andersen, Kin Lien Chee, Martin Jacob, Gert Frølund Pedersen, Thomas Kürner. Reverberation and Absorption in an Aircraft Cabin with the Impact of Passengers. accepted for publication at Antennas and Propagation Transactions, 2011.

[2] Claude Oestges and Bruno Clerckx. MIMO Wireless Communication: From Real-World Propagation to Space-Time Code Design. Academic Press, first edition, 2007.

[3] Emmeric Tanghe, Wout Joseph, Martine Lienard, Abdelmottaleb Nasr, Paul Stefanut, Luc Martens, and Pierre Degauque. Statistical Analysis of Multipath Clustering in an Indoor Office Environment. EURASIP Journal on Wireless Communications and Networking, page 16 pages, 2011.

[4] N. Czink. The Random-Cluster Model - A Stochastic MIMO Channel Model for Broadband Wireless Communication Systems of the 3rd Generation and Beyond. $\mathrm{PhD}$ thesis, Technische Universität Wien, Forschungszentrum Telekommunikation Wien, Wien, AT, 2007.

[5] 3rd Generation Partnership Project. Spatial Channel Model for MIMO Simulations . Technical report, 3GPP Technical Specification Group Radio Access Network, ref.: 3GPP TR 25.996 v8.0.0, online: www.3gpp.org., 2008.

[6] Heinrich Kuttruff. Room Acoustics. Spon Press, 2000, London.

[7] J. Bach Andersen, J. Ø. Nielsen, G. F. Pedersen, G. Bauch, and M. Herdin. Room Electromagnetics. IEEE Antennas and Propagation Magazine, 49, No. 2: pages 27-33, April 2007.

[8] Guidelines for limiting exposure to time-varying electric, magnetic, and electromagnetic fields (up to $300 \mathrm{GHz}$ ). Technical report, International Commission on Non-Ionizing Radiation Protection.

[9] G. Vermeeren, W. Joseph, C. Olivier, and L. Martens. Statistical multipath exposure of a human in a realistic electromagnetic environment. Health Physics, 94, no. 4: pages 345-354, April 2008.

[10] P. J. Dimbylow. Fine resolution calculations of SAR in the human body for frequencies up to $3 \mathrm{GHz}$. Physics In Medicine And Biology, 47: pages 2835-2846, 2002.

[11] Jianqing Wang, Osamu Fujiwara, Sachiko Kodera and Soichi Watanabe. FDTD calculation of whole-body average SAR in adult and child models for frequencies from $30 \mathrm{MHz}$ to $3 \mathrm{GHz}$. Physics In Medicine And Biology, 51: pages 4119-4127, 2006.

[12] Akimasa Hirata, Yoshio Nagaya, Fujiwara Osamu, Tomoaki Nagaoka, Soichi Watanabe. Correlation between Absorption Cross Section and Body Surface Area of Human for Far-Field Exposure at GHz Bands. Electromagnetic Compatibility, 2007. EMC 2007. IEEE International Symposium on, pages 1-4, July 2007.

[13] Nielsen J. Ø., Andersen J.B., Eggers P.C.F., Pedersen G.F., Olesen K., Suda H. Measurements of indoor $16 \times 32$ wideband MIMO channels at $5.8 \mathrm{GHz}$. IEEE Eighth International Symposium on Spread Spectrum Techniques and Applications, pages pages 864-868, August 2004.

[14] Fujimoto S, Watanabe T, Sakamoto A, Yukawa K, Morimoto K. Studies on the physical surface area of Japanese. 18. Calculation formulae in three stages over all ages. Nippon Eiseigaku Zasshi, 5: pages 443-450., 1968.

[15] Emmanuelle Conil, Abdelhamid Hadjem, Azeddine Gati, ManFai Wong and Joe Wiart. Influence of Plane-Wave Incidence Angle on Whole Body and Local Exposure at $2100 \mathrm{MHz}$. IEEE Transactions on Electromagnetic Compatibility, 53, No. 1: pages 48-52, 2011.
[16] Harald T. Friis. A note on a Simple Transmission Formula. Procedings of the I.R.E and Waves and Electrons, pages pages 254 256, May, 1946.

[17] E. Conil, A. Hadjem, F. Lacroux, M. F. Wong and J. Wiart. Variability analysis of SAR from $20 \mathrm{MHz}$ to $2.4 \mathrm{GHz}$ for different adult and child models using finite-difference timedomain. Physics In Medicine And Biology, 53: pages 15111525, 2008.

[18] Pejman Roshan, Jonathan Leary. 802.11 Wireless LAN Fundamentals. Cisco Press, Indianapolis, Indiana 46240 USA, December 23, 2003. 